\title{
Do Vitamin D Level and Dietary Calcium Intake Modify the Association Between Loop Diuretics and Bone Health?
}

\author{
Sadaf Oliai Araghi ${ }^{1,2} \cdot$ Jessica C. Kiefte-de Jong ${ }^{2,3} \cdot$ Katerina Trajanoska $^{1,2} \cdot$ Fjorda Koromani $^{1,2,7}$. \\ Fernando Rivadeneira ${ }^{1,2} \cdot$ M. Carola Zillikens ${ }^{1}$. Natasja M. van Schoor ${ }^{4}$. Lisette C. P. G. M. de Groot ${ }^{5}$. \\ M. Arfan Ikram ${ }^{2,6,7} \cdot$ André G. Uitterlinden ${ }^{1,2} \cdot$ Bruno H. Stricker $^{2} \cdot$ Nathalie van der Velde ${ }^{1,8}$
}

Received: 3 July 2019 / Accepted: 26 September 2019 / Published online: 14 October 2019

(c) The Author(s) 2019

\begin{abstract}
Loop diuretics (LD) may affect bone health by inhibiting renal calcium reuptake. However, whether vitamin D status and dietary calcium intake modify the association between $\mathrm{LD}$ and bone outcome is unclear. Therefore, this study aimed to evaluate whether vitamin $\mathrm{D}$ level or calcium intake modify the association between $\mathrm{LD}$ and various indices of bone health including bone mineral density (BMD) and Trabecular Bone Score (TBS). From The Rotterdam Study, a prospective population-based cohort study, we used data from 6990 participants aged > 45 year with a DXA scan (2002-2008), 6908 participants with femoral neck (FN)-BMD, 6677 participants with lumbar spine (LS)-BMD and 6476 participants with LS-TBS measurements. Use of LD was available from pharmacy dispensing records. Vitamin $\mathrm{D}(25(\mathrm{OH}) \mathrm{D})$ level was measured in serum, and dietary calcium intake was measured with a validated food frequency questionnaire. Almost eight percent of the participants used LD. The association between LD (past-users compared to never-users) and LS-TBS was significantly different by $25(\mathrm{OH})$ D concentrations $(P$ for interaction $=0.04)$. A significantly lower LS-TBS among LD past-users was observed for $25(\mathrm{OH})$ $\mathrm{D} \geq 50 \mathrm{nmol} / \mathrm{l}$ compared to $\leq 20$ and $20-50 \mathrm{nmol} / \mathrm{l}(\beta=-0.036,95 \% \mathrm{CI}-0.060 ;-0.013$ vs. $\beta=-0.012,95 \% \mathrm{CI}-0.036$; 0.013 and $\beta=-0.031,95 \% \mathrm{CI}-0.096 ; 0.034$, respectively). However, no other significant effect modification by $25(\mathrm{OH}) \mathrm{D}$ and dietary calcium intake was found in the associations between LD use and bone health outcomes $(P$-interaction $>0.13)$. This study suggests that the association between LD use and indices of bone health is not consistently modified by vitamin $\mathrm{D}$ or dietary calcium intake.
\end{abstract}

Keywords Loop diuretics $\cdot$ Bone mineral density $\cdot$ Bone geometry $\cdot$ Trabecular Bone Score

Electronic supplementary material The online version of this article (https://doi.org/10.1007/s00223-019-00621-1) contains supplementary material, which is available to authorized users.

Jessica C. Kiefte-de Jong

J.C.Kiefte@lumc.nl

1 Department of Internal Medicine, Erasmus University Medical Center, Rotterdam, The Netherlands

2 Department of Epidemiology, Erasmus University Medical Center, Rotterdam, The Netherlands

3 Department of Public Health and Primary Care, Leiden University Medical Center/LUMC Campus, The Hague, The Netherlands

4 Department of Epidemiology and Biostatistics, Amsterdam Public Health Research Institute, Amsterdam UMC, Vrije Universiteit Amsterdam, Amsterdam, The Netherlands
5 Division of Human Nutrition and Health, Wageningen University, Wageningen, The Netherlands

6 Department of Neurology, Erasmus Medical Center, Rotterdam, The Netherlands

7 Department of Radiology, Erasmus Medical Center, Rotterdam, The Netherlands

8 Section of Geriatric Medicine, Department of Internal Medicine, Amsterdam Public Health Research Institute, Amsterdam UMC, (University) of Amsterdam, Amsterdam, The Netherlands 


\section{Introduction}

In the past decade, recognition of the importance of food and drug interactions has been growing in clinical practice [1]. Especially in older people, however, more knowledge is needed because of the frequent use of medications and polypharmacy and higher risk of poor nutritional status [2]. Food-drug interactions may be relevant in older people using loop diuretics (LD). Diuretics are frequently prescribed in the treatment of heart failure and hypertension $[3,4]$, and they have been shown to influence calcium homeostasis and bone metabolism.

Thiazide diuretics have shown to have a protective effect in preserving bone mass and in decreasing the risk of fractures [5]. Yet, these effects have been shown to vary in relation to dosage, duration of treatment and do not last long after discontinuation of treatment [6-8]. In contrast, few studies have been carried out assessing the effect of loop diuretics (LD) on skeletal health. Some studies suggest that LD can have a negative impact on bone turnover by increasing urinary calcium excretion [9, 11-13], whereas others show no association between LD use and bone health on long-term use [6-13].

These conflicting findings may be the result of differences in calcium intake and vitamin D levels across the studied population, considering that bone mineral deposition/formation and bone resorption can be relatively normal as long as serum calcium and phosphate and calciotropic hormone levels like vitamin D and parathyroid hormone (PTH) are normal [14]. Vitamin D has a major role in calcium homeostasis through three mechanisms: (I) increased intestinal absorption of calcium, (II) reduced renal excretion of calcium by stimulating resorption of the distal tubules and (III) deposition and mobilization of calcium from bones [15]. An inverse association between LD use and serum 25(OH)D level has been reported before [16]. LD users have a lower concentration of $25(\mathrm{OH}) \mathrm{D}$ than non-users [17]. As a result, vitamin $\mathrm{D}$ deficiency may amplify any potential adverse effects of LD on bone health due to increased urinary calcium losses. Also, the use of LD may be harmful for bone health through increased plasma PTH and $1.25(\mathrm{OH})_{2} \mathrm{D}$ levels as a result of increased renal calcium losses [13]. Higher calcium loss may increase bone turnover resulting in a negative calcium balance. Furthermore, in case of very low external calcium supply from diet or supplementation, high levels of $1.25(\mathrm{OH})_{2} \mathrm{D}_{3}$ mobilize the bone calcium reservoir for serum calcium homeostasis at the (temporary) expense of bone mass and strength [18]. Accordingly, in people with a low calcium intake, the effect of LD on bone loss and increased fracture risk may be enhanced [10].

Therefore, in view of the frequent use of LD and a high prevalence of osteopenia and osteoporosis in the ageing population, in combination with a higher risk of malnutrition and vitamin D deficiency in older individuals, it is important to evaluate whether vitamin D level or calcium intake may modify the association between loop diuretics and various indices of bone health including bone mineral density (BMD) and trabecular bone score (TBS) in elderly population.

\section{Materials and Methods}

\section{Study Population}

This study was embedded in the first three cohorts of The Rotterdam Study (RS-I, RS-II and RS-III), an ongoing, population-based cohort study in Ommoord, a suburb of Rotterdam, the Netherlands [19]. Since January 1990, participants of 55 years and over were recruited for RS-I $(N=7983)$. In 2000, the study was extended to 3011 participants (RS-II). Later in 2006, the study was extended with a third cohort of participants of 45 years and older (RS-III). Overall response for all three cycles at baseline was $72 \%$ $(14,926$ of 20,744) [19]. Participants were interviewed at home by a trained research assistant, after which they were invited for a physical examination and dietary assessment at the research centre. More details on the main objectives, design and diagram of examination cycles of the Rotterdam Study (RS) have been published elsewhere [19]. Because of a possible persisting effect on bone, users of bisphosphonates were excluded from the study. For the current analysis, 6908 participants with data available for femoral neck (FN)-BMD, 6677 participants with lumbar spine (LS)-BMD and 6476 participants with LS-TBS were included from the fourth examination of the first cohort (RS-I-4, 2002-2004), the second examination of the second cohort (RS-II-2, 2004-2005) and the first examination of the third cohort (RS-III-1, 2006-2008) (Fig. 1).

\section{Dietary Intake and Serum 25(OHD) Level}

Dietary data were collected at baseline (between 1989 and 1993 in RS-I-1, between 2000 and 2001 in RS-II-1 and between 2006 and 2008 in RS-III-1) using a validated semiquantitative Food Frequency Questionnaire (FFQ) managed by a trained dietician, at the study centre [20, 21]. For RS-I-1 and RS-II-1, a two-stage 170-items FFQ was used (during first stage, participants mentioned on 170 food item, which foods they consumed at least twice a month in the preceding year, and in the second stage, dietician identified how often and in which amounts the foods were consumed). For RSIII-1, a one-stage 389-items FFQ was used. Dietary intake of nutrients (incl. total energy and dietary calcium intake) was determined using the Dutch Food Composition Tables 


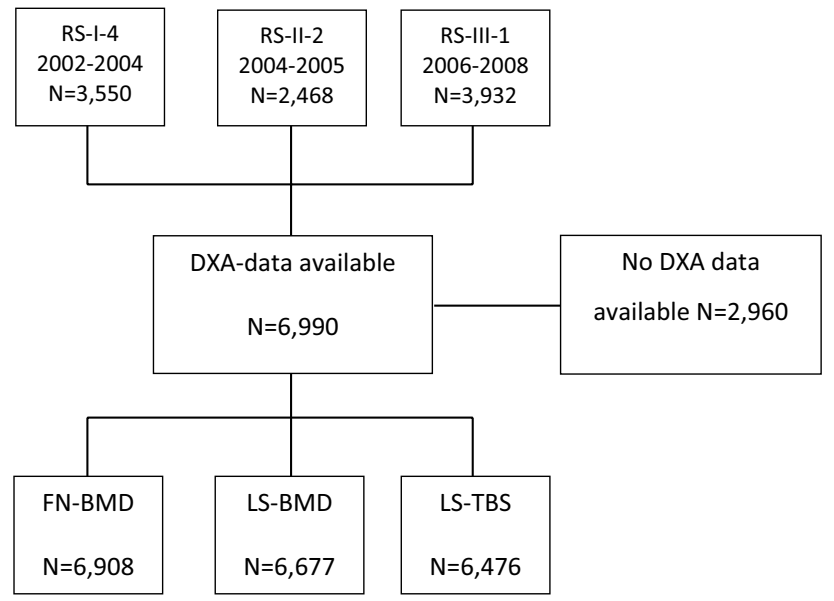

Fig. 1 Flow-chart of sub-cohorts included in the study

(NEVO) from 1993, 2001 and 2006, using standardized portion sizes [20, 22]. Intake of calcium was adjusted for energy corresponding to the residual method [23]. Serum 25(OH) $\mathrm{D}$ was measured in the blood collected at the same time as dietary data, between 1990 and 2008 using electrochemiluminescence immunoassay (COBAS, Roche Diagnostics $\mathrm{GmbH}$ ). The sensitivity of the test was $10 \mathrm{nmol} / \mathrm{L}$, the range of serum $25(\mathrm{OH}) \mathrm{D}$ concentrations was from $7.5 \mathrm{nmol} / \mathrm{L}$ to $175 \mathrm{nmol} / \mathrm{L}$, the within-run accuracy was less than $7.8 \%$, and the intermediate precision accuracy was less than $13.1 \%$ [24, $25]$. Vitamin D deficiency was defined as a serum $25(\mathrm{OH}) \mathrm{D}$ level $\leq 50 \mathrm{nmol} / \mathrm{L}$ according to the current recommendations for older adults aged $>70$ years by the Institute of Medicine and the Dutch Health Council [26]. In addition, we used also a vitamin $\mathrm{D}$ deficiency threshold $<20 \mathrm{nmol} / \mathrm{L}$ in our analysis and showed the stratification analysis in categories of $\leq 20 \mathrm{nmol} / \mathrm{L}$, between $20-50 \mathrm{nmol} / \mathrm{L}$ and $\geq 50 \mathrm{nmol} / \mathrm{L}$.

\section{Loop Diuretics}

As of 1st January 1991, pharmacy records of prescriptions were collected via all pharmacies in the Ommoord region with details on product name, ATC code, number of tablets/ capsules in each prescription, and prescribed daily number [27]. LD use (ATC code C03C) was determined from baseline to the date of the DXA scan [19] and defined in three different categories: current-users, past-users and neverusers. Current-users were defined when the participant had a drug exposure period between the date of the DXA scan and 120 days prior to the performed DXA scan. Past-users were defined when the participant had a drug exposure period more than 120 days prior to the performed DXA scan. In addition, if the participants had no drug exposure from baseline till the DXA scan, the participants were considered as never-users. The duration of LD use among current-users was categorized into $1-120$ days, $120-365$ days and more than 365 days. Never-use of LD was used as the reference category.

\section{Assessment of Co-variables}

Co-variables related to lifestyle, body composition and socioeconomic status (SES) were included. Weight $(\mathrm{kg})$ and height $(\mathrm{cm})$ were measured at study entry. BMI was calculated as weight divided by height squared $\left(\mathrm{kg} / \mathrm{m}^{2}\right)$. During home interviews physical activity (PA) was assessed by means of the Zutphen Physical Activity Questionnaire [28]. Metabolic equivalent of tasks were calculated (MET hours/ week) according to time spent in categories of light, moderate and vigorous activity [28]. Socioeconomic status variables (i.e. educational level and income level), smoking (yes/ no), pack-years, use of alcohol, prevalence of coronary heart diseases (CHD), stroke and diabetes mellitus (DM) were assessed by home interview. Also the use of bisphosphonates was determined using pharmacy dispensing records in the same way as the use of LD. Serum calcium, magnesium and sodium were measured in blood samples by the Department of Clinical Chemistry of the Erasmus Medical Center using the Roche/Hitachi cobas c501 analyzer (Roche Diagnostics, Indianapolis, IN, USA).

\section{Outcome Assessment}

Femoral neck and lumbar spine BMD was measured at RS-I4, RS-II-2 and RS-III-1 (between 2002 and 2008) by dualenergy X-ray absorptiometry (DXA) using a ProdigyTM fan-beam densitometer (GE Lunar Corp, Madison, WI, USA for all participants [19]. The DXA-derived trabecular bone score (TBS), which is measured in the Lumbar Spine (LSTBS), is a grey-level texture measurement that correlates with 3D parameters of bone microarchitecture, connectivity density, trabecular separation and trabecular number [29]. LS-TBS predicts future fractures (all type) independent of areal BMD [30]. Moreover, recent studies have shown that LS-TBS may be an applicable measure of trabecular bone integrity to study in regard to lifestyle factors that are adaptable, such as a dietary intake $[31,32]$. LS-TBS was derived from the same lumbar DXA scans that BMD was obtained from and it was analysed using TBS iNsight software (version 1.9; Medimaps, Geneva, Switzerland) at the Bone Disease Unit of the University of Lausanne (Lausanne, Switzerland). LS-TBS was calculated for a subgroup of RS-I-4, RS-II-2 and RS-III- 1 and represents the mean value of the individual vertebral measurements from L1 to L4. Subjects with a BMI higher than $37 \mathrm{~kg} / \mathrm{m}^{2}$ were excluded from the study since LS-TBS measurements in morbidly obese persons are not accurate. Furthermore, the LS-TBS was standardized according to sex using the residual method, due to 
technical differences between LS-TBS across sexes. The method of LS-TBS calculation has been described in detail elsewhere [32].

\section{Statistical Analyses}

First, for all variables normal distribution was examined by visual check of histograms. When necessary, data were log transformed. Linear regression analysis (cross-sectional) was used to assess the association between LD use (past-, current-use and the duration of 1-120 days, 120-365 days and $>365$ days) and FN-BMD, LS-BMD and LS-TBS. All the analyses were adjusted for age and sex and cohort (model 1). Thereafter, the co-variables were added additionally to model 2, based on literature relevance as well as principles of causal inference combined with the change-in-effect-criterion of $\geq 10 \%[33,34]$. Potential confounders were BMI, smoking (pack-years), alcohol intake (g/day), SES (education and job), total physical activity (MET hours/week) and comorbidities (prevalence of CHD, stroke and DM). For the analysis of the duration (continuous), the model was additionally adjusted for the past-users. An earlier study showed that in subjects with osteopenia/normal BMD levels, TBS is significantly associated with vertebral fractures [35]. Even though BMD and TBS are correlated, they present different aspects of bone health [35]. For that reason, as sensitivity analyses, we wanted to assess how the association between LD and BMD may depend on measures of bone architecture (i.e. TBS) and how a potential association between LD and TBS is dependent on BMD. So, the association between LD and FN-BMD and LS-BMD was additionally adjusted for LS-TBS and vice versa (model 3). In addition, in all analyses, sensitivity analyses were performed with additional adjustment for serum vitamin $\mathrm{D}$, season of blood collection of vitamin D [winter (September till end of February) and summer (March till end of August)], serum calcium, magnesium and sodium concentrations.

To assess whether effect modification by serum 25(OHD) level or dietary calcium intake was present in the association of LD (ever-use) on FN-BMD, LS-BMD and LS-TBS, we evaluated the effect modification by serum $25(\mathrm{OH}) \mathrm{D}$ level and energy adjusted dietary calcium intake on FN-BMD, LS-BMD and LS-TBS in model 2 ( $P$ for interaction $<0.10$ ), since the beta did not changed after additional adjustment in model 3. Thereafter, we stratified the analyses of LD use (LD never-use, current-use and past-use with neveruse as reference category) and bone outcomes according to serum $25(\mathrm{OH}) \mathrm{D}$ level, in subsequent categories of $\leq 20$, between $20-50$ and $\geq 50 \mathrm{nmol} / \mathrm{L}$ ) and energy adjusted dietary intake of calcium in following categories: $\leq 950 \mathrm{mg} /$ day, $950-1200 \mathrm{mg} /$ day and $\geq 1200 \mathrm{mg} /$ day. The association between LD and LS-TBS, LS-BMD and FN-BMD was also evaluated by sex through effect modification.
To account for missing data in co-variables (varied from 1.6 to $66.8 \%$ ), we used a multiple imputation approach ( $n=10$ imputations, 10 iterations). Briefly, as described by Sterne et al, multiple imputation is created on the correlation between each variable and missing values with other participant characteristics [36]. Linear regression analyses were then separately accomplished in each of the 10 datasets [36]. Betas were pooled by taking the average of the effect sizes of the 10 imputed datasets. The pooled standard errors and respective 95\% Confidence Intervals (CI) were then calculated by using Rubin's rules [37]. For details of the multiple imputation, see supplement Tables S1 and S2. The statistical software package of SPSS 24.0 was used for the statistical analyses (SPSS Inc., Chicago, Illinois, USA). For all the analyses except the effect-modification analysis, $p$ values of $<0.05$ were considered statistically significant.

\section{Results}

\section{Population Characteristics}

Baseline characteristics are presented for the total population, LD users and non-users (Table 1) and baseline characteristics before and after multiple imputation are shown in Table S2. The median age, on the DXA scan date, was 65.0 years [57.0-99.0 IQR] for the total population $(n=6990)$. Of the total population, 7.8\% $(n=543)$ ever used LD from baseline till the DXA scan with a mean duration of 51.2 days (325.1 SD). The median age of the $543 \mathrm{LD}$ users was 77.0 years [52.0-99.0 IQR] and 64.0 years [51.0-97.0 IQR] for the LD non-users (Table 1).

The median serum 25(OH)D level of the LD users was $42.3 \mathrm{nmol} / \mathrm{L}$ [28.8-62.3 IQR] and for LD non-users, $55.4 \mathrm{nmol} / 1$ [37.2-76.7 IQR]. LD users showed a significantly lower serum 25(OH)D level than non-users $(p<0.001)$. The mean dietary calcium intake of the LD users was $1189 \mathrm{mg} /$ day (394 SD) and $1121 \mathrm{mg} /$ day (394 SD) for LD non-users. Results of the linear regression analysis of LD use and indices of bone health are shown in Table 2 and discussed below.

\section{Loop Diuretics and LS-TBS}

Compared to LD never-use, current-use of LD was only associated with LS-TBS in the crude model $(\beta=-0.018$, $95 \% \mathrm{CI}-0.034 ;-0.001)$ and past-use of LD was associated with lower LS-TBS, in model $1(\beta=-0.031,95 \% \mathrm{CI}$ $-0.044 ;-0.017)$. Adjustment for covariates in model 2 attenuated the association somewhat whereby the effect size was 30\% lower. The analysis of categories of the duration of LD use among users and LS-TBS showed no significant associations (Table 2). 
Table 1 Characteristics total population, users and non-users of LD

Total population $(n=6990) \quad$ LD ever-users $(n=543) \quad$ LD never-users $(n=6447) \quad p$ value LD everversus never-users

\begin{tabular}{|c|c|c|c|c|}
\hline Age (years) ${ }^{\mathrm{b}}$ & $65.0[57.0-99.0]$ & $77.0[52.0-99.0]$ & $64.0[51.0-97.0]$ & $<0.001^{*}$ \\
\hline Sex (\%women) & $3985(57.0)$ & $309(56.9)$ & $3675(57.0)$ & 0.92 \\
\hline BMI $\left(\mathrm{kg} / \mathrm{cm}^{2}\right)^{\mathrm{a}}$ & $27.6(4.1)$ & $28.9(4.7)$ & $27.5(4.7)$ & $0.002 *$ \\
\hline Cohort (\%) & & & & $<0.001^{*}$ \\
\hline RS-I & $2799(40.0)$ & $437(80.5)$ & $2360(36.6)$ & \\
\hline RS-II & $980(14.0)$ & $62(11.4)$ & $918(14.2)$ & \\
\hline RS-III & $3211(46.0)$ & $44(8.1)$ & $3167(49.1)$ & \\
\hline Loop diuretics use (\%ever use) & $543(7.8)$ & $543(100)$ & 0 & NA \\
\hline Duration (days) ${ }^{\mathrm{a}}$ & NA & 194 [40-893] & 0 & \\
\hline $25(\mathrm{OH}) \mathrm{D}(\mathrm{nmol} / \mathrm{l})^{\mathrm{b}}$ & $54.4[36.5-76.0]$ & $42.3[28.8-62.3]$ & $55.4[37.2-76.7]$ & $<0.001^{*}$ \\
\hline Cutoff $(\%)$ & & & & $<0.001 *$ \\
\hline$<50 \mathrm{nmol} / \mathrm{L}$ & $2580(44.2)$ & $247(45.5)$ & $2333(36.2)$ & 0.41 \\
\hline$>50 \mathrm{nmol} / \mathrm{L}$ & $3257(55.8)$ & $156(28.7)$ & $3101(48.1)$ & \\
\hline \multicolumn{5}{|l|}{ Season measurement (\%) } \\
\hline Spring & $1928(27.6)$ & $147(27.0)$ & $1854(28.8)$ & \\
\hline Summer & $1143(16.4)$ & $82(15.1)$ & $1107(17.2)$ & \\
\hline Autumn & $2219(31.7)$ & $177(32.5)$ & $2126(33.0)$ & \\
\hline Winter & $1432(20.5)$ & $138(25.4)$ & $1360(21.0)$ & \\
\hline 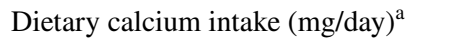 & 1126.5 (388.9) & $1189.2(394.1)$ & $1120.8(394.1)$ & 0.81 \\
\hline \multicolumn{5}{|l|}{ Tertiles (\%) } \\
\hline$<950.0 \mathrm{mg} /$ day & $1691(24.2)$ & $103(19.0)$ & $1588(24.6)$ & \\
\hline $950-1200 \mathrm{mg} /$ day & $1504(21.5)$ & $120(22.1)$ & $1384(21.5)$ & \\
\hline$>1200 \mathrm{mg} /$ day & $1795(25.7)$ & $186(34.3)$ & $2609(25.0)$ & \\
\hline${\text { Lumbar spine } \mathrm{TBS}^{\mathrm{a}}}$ & $1.24(0.13)$ & $1.17(0.12)$ & $1.25(0.12)$ & 0.32 \\
\hline $\begin{array}{l}\text { Femoral neck bone mass density (g/ } \\
\left.\mathrm{cm}^{2}\right)^{\mathrm{a}}\end{array}$ & $0.91(0.15)$ & $0.86(0.15)$ & $0.91(0.15)$ & 0.98 \\
\hline $\begin{array}{l}\text { Lumbar spine bone mass density (g/ } \\
\left.\mathrm{cm}^{2}\right)^{\mathrm{a}}\end{array}$ & $1.14(0.21)$ & $1.15(0.22)$ & $1.14(0.20)$ & 0.40 \\
\hline \multicolumn{5}{|l|}{ Smoking (\%) } \\
\hline Never smoker & $2080(29.8)$ & $157(28.9)$ & $1921(29.8)$ & $0.003^{*}$ \\
\hline Current smoker & $1358(19.4)$ & $58(10.6)$ & $1302(20.2)$ & \\
\hline Former, non-smoker & $3506(50.2)$ & $319(58.8)$ & $3185(49.4)$ & \\
\hline Pack-years $^{\mathrm{a}}$ & $9.4(18.6)$ & $20.3(28.0)$ & $8.5(28.0)$ & $<0.001^{*}$ \\
\hline \multicolumn{5}{|l|}{ Education category (\%) } \\
\hline Low education & 3575 (51.6) & $319(58.7)$ & $3256(50.9)$ & $<0.001^{*}$ \\
\hline Higher education & $3353(48.4)$ & $219(40.3)$ & $3134(49.1)$ & \\
\hline Alcohol intake (g/day) ${ }^{\mathrm{b}}$ & $7.3[0.8-20.0]$ & $3.1[0.1-15.7]$ & $7.9[0.9-20.0]$ & 0.11 \\
\hline PA (MET h/week) ${ }^{\mathrm{b}}$ & 70.5 [39.4-103.9] & 70.8 [47.6-100.1] & $70.4[38.5-104.4]$ & 0.84 \\
\hline Energy intake $(\mathrm{kcal} / \mathrm{day})^{\mathrm{a}}$ & $2176.6(710.2)$ & $1956.9(533.8)$ & $2195.2(533.8)$ & $<0.001^{*}$ \\
\hline Comorbidities (\%yes) & $1112(15.9)$ & $150(27.6)$ & $963(14.9)$ & $<0.001^{*}$ \\
\hline CHD & $315(4.5)$ & $69(12.7)$ & $246(3.8)$ & \\
\hline Stroke & $129(1.8)$ & $13(2.4)$ & $116(1.8)$ & \\
\hline $\mathrm{DM}$ & $790(11.3)$ & $98(18.0)$ & $691(10.7)$ & \\
\hline
\end{tabular}

$* p$ value $<0.05$

${ }^{\mathrm{a}}$ Mean (SD)

${ }^{\mathrm{b}}$ Median (IQR)

$\%$ percentage 
Table 2 Linear regression of the use of LD and FN-BMD and LS-TBS in RS-I-4, RS-II-2 \& RS-III-1

\begin{tabular}{|c|c|c|c|}
\hline & $\begin{array}{l}\text { Model } 1 \\
\beta(95 \% \mathrm{CI})\end{array}$ & $\begin{array}{l}\text { Model } 2 \\
\beta(95 \% \mathrm{CI})\end{array}$ & $\begin{array}{l}\text { Model } 3 \\
\beta(95 \% \mathrm{CI})\end{array}$ \\
\hline \multicolumn{4}{|l|}{$L S-T B S(6476)^{\mathrm{a}}$} \\
\hline LD never-use (5972) & Reference & Reference & Reference \\
\hline LD past-use $(n=310)$ & $-0.031[-0.044 ;-0.017]^{*}$ & $-0.019[-0.033 ;-0.006]^{*}$ & $-0.025[-0.038 ;-0.012]^{*}$ \\
\hline LD current-use $(n=194)$ & $-0.018[-0.034 ;-0.001]^{*}$ & $-0.011[-0.028 ; 0.006]$ & $-0.015[-0.032 ; 0.002]$ \\
\hline \multicolumn{4}{|l|}{ Duration of LD-use categories ${ }^{\mathrm{b}}$} \\
\hline Never-users $(n=5972)$ & Reference & Reference & Reference \\
\hline Current-users $1-120$ days $(n=37)$ & $-0.008[-0.027 ; 0.011]$ & $-0.0005[-0.043 ; 0.042]$ & $-0.003[-0.044 ; 0.039]$ \\
\hline Current-users $121-365$ days $(n=27)$ & $-0.026[-0.048 ; 0.003]$ & $-0.026[-0.070 ; 0.018]$ & $-0.041[-0.084 ; 0.002]$ \\
\hline Current-users $>365$ days $(n=310)$ & $-0.019[-0.039 ; 0.001]$ & $-0.010[-0.031 ; 0.010]$ & $-0.013[-0.032 ; 0.007]$ \\
\hline \multicolumn{4}{|l|}{$L S-B M D(n=6677)$} \\
\hline LD never-use (6146) & Reference & Reference & Reference \\
\hline LD past-use $(n=321)$ & $0.048[0.026 ; 0.071]^{*}$ & $0.029[0.006 ; 0.052]^{*}$ & $0.038[0.016 ; 0.060]^{*}$ \\
\hline LD current-use $(n=210)$ & $0.037[0.010 ; 0.065]^{*}$ & $0.021[-0.008 ; 0.050]$ & $0.025[-0.003 ; 0.052]$ \\
\hline \multicolumn{4}{|l|}{ Duration of LD-use categories ${ }^{\mathrm{b}}$} \\
\hline Never-users $(n=6146)$ & Reference & Reference & Reference \\
\hline Current-users $1-120$ days $(n=41)$ & $0.020[-0.040 ; 0.081]$ & $0.012[-0.059 ; 0.083]$ & $0.014[-0.054 ; 0.082]$ \\
\hline Current-users $121-365$ days $(n=32)$ & $0.072[0.004 ; 0.140]^{*}$ & $0.068[-0.003 ; 0.139]$ & $0.075[0.007 ; 0.143]^{*}$ \\
\hline Current-users $>365$ days $(n=137)$ & $0.034[0.0004 ; 0.068]^{*}$ & $0.012[-0.022 ; 0.047]$ & $0.016[-0.017 ; 0.049]$ \\
\hline \multicolumn{4}{|l|}{$F N-B M D(n=6908)$} \\
\hline LD never-use (6376) & Reference & Reference & Reference \\
\hline LD past-use $(n=319)$ & $0.016[0.001 ; 0.031]^{*}$ & $0.006[-0.009 ; 0.021]$ & $0.010[-0.005 ; 0.025]$ \\
\hline LD current-use $(n=213)$ & $0.006[-0.012 ; 0.024]$ & $-0.006[-0.025 ; 0.013]$ & $-0.006[-0.024 ; 0.013]$ \\
\hline \multicolumn{4}{|l|}{ Duration of LD-use categories ${ }^{\mathrm{b}}$} \\
\hline Never-users $(n=6376)$ & Reference & Reference & Reference \\
\hline Current-users $1-120$ days $(n=44)$ & $0.020[-0.019 ; 0.058]$ & $0.024[-0.021 ; 0.069]$ & $0.021[-0.025 ; 0.067]$ \\
\hline Current-users $121-365$ days $(n=32)$ & $0.035[-0.011 ; 0.080]$ & $0.019[-0.028 ; 0.066]$ & $0.023[-0.023 ; 0.069]$ \\
\hline Current-users $>365$ days $(n=137)$ & $-0.005[-0.027 ; 0.018]$ & $-0.019[-0.042 ; 0.003]$ & $-0.018[-0.040 ; 0.004]$ \\
\hline
\end{tabular}

Model 1: sex, age, cohort. Model 2: additional adjusted for BMI, alcohol, smoking, SES, PA and comorbidities (for duration of LD use additional adjusted for past-users). Model 3: + LS-TBS (for the analysis of FN-BMD)/FN-BMD and LS-BMD (for the analysis of LS-TBS)

${ }^{*} p<0.05$

${ }^{\text {a }}$ Standardized according to sex by residual method

${ }^{\mathrm{b}}$ Past-users depicted in the rows above

\section{Loop Diuretics and LS-BMD}

Current-use of LD in 210 participants was associated with significantly higher LS-BMD compared to never-use of LD in the crude model $(\beta=0.037,95 \%$ CI $0.010 ; 0.065)$. Pastuse of LD was associated with higher LS-BMD compared to never-use of LD in the crude model, model 2 and fully adjusted model (model 3$)(\beta=0.048,95 \%$ CI $0.026 ; 0.071$, $\beta=0.029,95 \%$ CI $0.006 ; 0.052$ and $\beta=0.038,95 \%$ CI 0.016 ; 0.060 ). In the analyses of the duration of LD use, currentuse of LD between 121 and 365 days showed a significantly higher LS-BMD in fully adjusted model $(\beta=0.075,95 \%$ CI 0.007; 0.143 Table 2).

\section{Loop Diuretics and FN-BMD}

LD current-users showed no significant association with FNBMD compared to never-use of LD. However, past-use of LD showed a significantly higher FN-BMD only in the crude model $(\beta=0.016,95 \%$ CI $0.001 ; 0.031$, Table 2$)$.

Additional adjustment for serum vitamin $\mathrm{D}$, season of blood collection of vitamin $\mathrm{D}$, serum calcium, magnesium and sodium did not change the results (data not shown).

Furthermore, we evaluated the association between LD and LS-TBS, LS-BMD and FN-BMD by sex and found no evidence that the association between LD and LS-TBS was significantly different according to sex ( $P$-interaction $=0.83$ ). However, the association between LD and 
LS-BMD and FN-BMD was significantly different according to sex $(P$-interaction for both $=0.04)$ (Supplemental table S3).

\section{Serum 25(OH)D Level and Dietary Calcium Intake in the Association of the Use of Loop Diuretics and FN-BMD, LS-BMD and LS-TBS}

Effect-modification analyses of serum 25(OH)D level and dietary calcium intake in the association between LD use on FN-BMD and LS-TBS are shown in Tables 3 and 4. $P$ value for interaction terms varied from 0.04 to 0.99 . Serum $25(\mathrm{OH}) \mathrm{D}$ level was an effect modifier in the association between LD use and LS-TBS ( $P$ for interaction $=0.04)$. After stratification in model 2, the group of serum $25(\mathrm{OH})$ D level $\geq 50 \mathrm{nmol} / \mathrm{L}$ showed a significantly lower LS-TBS compared to serum $25(\mathrm{OH}) \mathrm{D} \leq 20 \mathrm{nmol} / \mathrm{L}$ and between 20 and $50 \mathrm{nmol} / \mathrm{L}$ for LD past-use $(\beta=-0.036,95 \% \mathrm{CI}$ $-0.060 ;-0.013$ vs. $\beta=-0.012,95 \% \mathrm{CI}-0.036 ; 0.013$ and $\beta=-0.031,95 \% \mathrm{CI}-0.096 ; 0.034$, respectively, Table 3 ).

There was no effect modification by serum $25(\mathrm{OH}) \mathrm{D}$ level on the association between LD use and FN-BMD and LS-BMD (Table 3). After stratification, for the analysis of the association between LD and LS-BMD, participants with serum 25(OH)D level between 20 and $50 \mathrm{nmol} / \mathrm{L}$ had a significantly higher LS-BMD compared to participants with serum $25(\mathrm{OH}) \mathrm{D}$ level $\leq 20$ and $\geq 50 \mathrm{nmol} / \mathrm{l}$ for LD currentuse; however, there was no significant interaction $(P$ for interaction $=0.30)($ Table 3$)$.

No significant effect modification by dietary calcium intake was observed on the association between use of LD and FN-BMD, LS-BMD and LS-TBS (Table 4). After stratification of dietary calcium intake in categories of intake $\leq 950,950-1200$ and $\geq 1200 \mathrm{mg} /$ day, no significant associations were found with FN-BMD, LS-BMD and LS-TBS (Table 4).

\section{Discussion}

In this study, we observed a modest increase in BMD of the lumbar spine and a modest decrease in TBS in LD pastusers, and therefore our study conclude that, if any, LD use does not have a strong association with bone health, in a population with a high calcium intake. Furthermore, this study found effect modification by serum $25(\mathrm{OH}) \mathrm{D}$ level in the association between LD and LS-TBS. However, after stratification on serum $25(\mathrm{OH}) \mathrm{D}$, no consistent findings were

Table 3 Linear regression of LD use (yes/no) on FN-BMD and LS-TBS for 25(OH)D level in categories $(\leq 20,20-50$ and $\geq 50 \mathrm{nmol} / \mathrm{l})$ in model 2

$\begin{array}{llll}25(\mathrm{OH}) \mathrm{D} \leq 20 \mathrm{nmol} / \mathrm{l}(n=302) & 25(\mathrm{OH}) \mathrm{D} 20-50 \mathrm{nmol} / \mathrm{l}(n=2240) & 25(\mathrm{OH}) \mathrm{D} \geq 50 \mathrm{nmol} / 1 & \begin{array}{l}p \text { value } \\ \text { interaction } \\ \beta(95 \% \mathrm{CI})\end{array} \\ & \beta(95 \% \mathrm{CI}) & \beta(95 \% \mathrm{CI}) & \text { term }\end{array}$

$L S-T B S(6476)^{\mathrm{a}}$

LD never-use

Reference Refence

LD past-use $(n=310)$

$-0.012[-0.036 ; 0.013]$

$-0.031[-0.096 ; 0.034]$

Reference

$0.04 * *$

LD current-use $(n=194)$

$0.001[-0.036 ; 0.039]$

$-0.029[-0.099 ; 0.041]$

$-0.036[-0.060 ;-0.013]^{*}$

$25(\mathrm{OH}) \mathrm{D} \leq 20 \mathrm{nmol} / \mathrm{l}(n=287)$

25(OH)D $20-50 \mathrm{nmol} / \mathrm{l}(n=2146)$

$-0.014[-0.046 ; 0.017]$

$\beta(95 \% \mathrm{CI})$

$\beta(95 \% \mathrm{CI})$

$25(\mathrm{OH}) \mathrm{D} \geq 50 \mathrm{nmol} / 1$

$p$ value $(n=3108)$

$\beta(95 \% \mathrm{CI})$

$L S-B M D(n=6677)$

LD never-use Reference

LD past-use $(n=321)$

0.079 [-0.054; 0.148]

Reference

Reference

0.30

LD current-use $(n=210)$

$-0.052[-0.157 ; 0.053]$

0.003 [-0.034; 0.041$]$

0.038 [-0.003; 0.080]

$25(\mathrm{OH}) \mathrm{D} \leq 20 \mathrm{nmol} / \mathrm{l}(n=267)$

0.044 [0.002; 0.087]*

0.039 [-0.016;0.095]

$25(\mathrm{OH}) \mathrm{D} 20-50 \mathrm{nmol} / \mathrm{l}(n=2054)$

$25(\mathrm{OH}) \mathrm{D} \geq 50 \mathrm{nmol} / \mathrm{l}$ $(n=3056)$

$\beta(95 \% \mathrm{CI})$

$\beta(95 \% \mathrm{CI})$

$\beta(95 \% \mathrm{CI})$

$F N-B M D(n=6908)$

LD never-use

Reference

Reference

Reference

0.13

LD past-use $(n=319)$

0.027 [-0.037; 0.092]

$-0.006[-0.031 ; 0.019]$

0.008 [- 0.020; 0.036]

LD current-use $(n=213)$

-0.022 [-0.089; 0.045]

$-0.004[-0.031 ; 0.023]$

$0.015[-0.021 ; 0.051]$

${ }^{\text {a }}$ Standardized according to sex by residual method

$* p<0.05 ; * * p<0.10$ 
Table 4 Linear regression of LD use (yes/no) on FN-BMD and LS-TBS for dietary calcium intake categories ( $\leq 950,950-1200$ and $\geq 1200 \mathrm{mg}$ / day) in model 2

$\begin{array}{lll}\begin{array}{l}\text { Dietary calcium intake } \\ \leq 950 \mathrm{mg} / \text { day }(n=1822)\end{array} & \begin{array}{l}\text { Dietary calcium intake } \\ 950-1,200 \mathrm{mg} / \text { day } \\ (n=1600)\end{array} & \begin{array}{l}\text { Dietary calcium intake } \\ \geq 1200 \mathrm{mg} / \text { day }(n=1875)\end{array} \\ \beta(95 \% \mathrm{CI}) & \beta(95 \% \mathrm{CI}) & \beta(95 \% \mathrm{CI})\end{array}$

\begin{tabular}{|c|c|c|c|c|}
\hline \multicolumn{5}{|l|}{$L S-T B S(6476)^{\mathrm{a}}$} \\
\hline LD never-use & Reference & Reference & Reference & \multirow[t]{2}{*}{0.58} \\
\hline $\begin{array}{l}\text { LD past-use }(n=310) \text { (yes/ } \\
\text { no) }\end{array}$ & $-0.018[-0.047 ; 0.010]$ & $-0.022[-0.050 ; 0.005]$ & $-0.015[-0.037 ; 0.007]$ & \\
\hline \multirow{3}{*}{$\begin{array}{l}\text { LD current-use }(n=194) \\
\quad(\text { yes/no) }\end{array}$} & $-0.009[-0.044 ; 0.025]$ & $0.007[-0.028 ; 0.043]$ & $-0.015[-0.043 ; 0.013]$ & \\
\hline & $\begin{array}{l}\text { Dietary calcium intake } \\
\leq 950 \mathrm{mg} / \text { day }(n=1732)\end{array}$ & $\begin{array}{l}\text { Dietary calcium intake } \\
950-1200 \mathrm{mg} / \text { day }(n=1548)\end{array}$ & $\begin{array}{l}\text { Dietary calcium intake } \\
\geq 1200 \mathrm{mg} / \text { day }(n=1838)\end{array}$ & \multirow[t]{2}{*}{$p$ value interaction term } \\
\hline & $\beta(95 \% \mathrm{CI})$ & $\beta(95 \% \mathrm{CI})$ & $\beta(95 \% \mathrm{CI})$ & \\
\hline \multicolumn{5}{|l|}{$L S-B M D(n=6677)$} \\
\hline LD never-use & Reference & Reference & Reference & \multirow[t]{2}{*}{0.87} \\
\hline $\begin{array}{l}\text { LD past-use }(n=321) \text { (yes/ } \\
\text { no) }\end{array}$ & $0.017[-0.034 ; 0.068]$ & $0.039[-0.008 ; 0.086]$ & $0.026[-0.012 ; 0.063]$ & \\
\hline \multirow{3}{*}{$\begin{array}{l}\text { LD current-use }(n=210) \\
\quad(\text { yes } / \text { no })\end{array}$} & $0.038[-0.023 ; 0.100]$ & $0.045[-0.016 ; 0.106]$ & $0.016[-0.031 ; 0.064]$ & \\
\hline & $\begin{array}{l}\text { Dietary calcium intake } \\
\leq 950 \mathrm{mg} / \text { day }(n=1695)\end{array}$ & $\begin{array}{l}\text { Dietary calcium intake } \\
950-1200 \mathrm{mg} / \text { day }(n=1509)\end{array}$ & $\begin{array}{l}\text { Dietary calcium intake } \\
\geq 1200 \mathrm{mg} / \text { day }(n=1771)\end{array}$ & \multirow[t]{2}{*}{$p$ value interaction term } \\
\hline & $\beta(95 \% \mathrm{CI})$ & $\beta(95 \% \mathrm{CI})$ & $\beta(95 \% \mathrm{CI})$ & \\
\hline \multicolumn{5}{|l|}{$F N-B M D(n=6908)$} \\
\hline LD never-use & Reference & Reference & Reference & \multirow[t]{3}{*}{0.99} \\
\hline $\begin{array}{l}\text { LD past-use }(n=319) \text { (yes/ } \\
\text { no) }\end{array}$ & $0.001[-0.032 ; 0.034]$ & $0.087[-0.023 ; 0.039]$ & $0.008[-0.017 ; 0.034]$ & \\
\hline $\begin{array}{l}\text { LD current-use }(n=213) \\
\quad(\text { yes } / \text { no) }\end{array}$ & $-0.003[-0.043 ; 0.037]$ & $0.024[-0.015 ; 0.064]$ & $-0.025[-0.057 ; 0.007]$ & \\
\hline
\end{tabular}

${ }^{*} p<0.05$

${ }^{\text {a }}$ Standardized according to sex by residual method

found in the association between LD use and bone outcomes, suggesting no strong modifying effect of serum $25(\mathrm{OH}) \mathrm{D}$ on these associations. Additionally, no effect modification was found by dietary calcium intake.

In our study, current LD use showed a negative association with LS-TBS, however not significant.

In contrast to the findings of the association between LD and LS-BMD, we found that a history of LD was associated with a decreased LS-TBS if LD was used in the past. To our knowledge, there have been no studies on LD use and LS-TBS. TBS is a measurement related to bone microarchitecture which provides skeletal information that is not captured from the standard BMD measurements [31]. Also, TBS might be an appropriate measure to study in regard to lifestyle factors that are adaptable, such as dietary intake $[31,32]$. Other studies have reported lower TBS among individuals with primary hyperparathyroidism [38]. And as mentioned, LDs increase the plasma PTH and 1.25(OH)2D levels as a result of increased renal calcium losses [11]. Thus, a possible explanation for our finding is that LS-TBS may be decreased by secondary hyperparathyroidism caused by long-time LD use [13, 39]. However, we could not confirm this possible pathway in our current study, since we do not have the availability of serum PTH. Other explanation for the opposite direction between BMD and TBS is due to potential residual confounding (i.e. body composition and health status) in the association between LD and TBS. Further studies are needed to explore the association between LD and overall bone health, especially TBS.

Additionally, LD use was not associated with a lower BMD. In contrast, it has been shown that LD increases renal calcium excretion [8-11], with a potential negative effect on BMD [10, 13, 38]. We expected that LD use would result in a lower FN-BMD and LS-BMD, because treatment with LD may induce secondary hyperparathyroidism with raised bone resorption resulting in a lower BMD [39, 40]. This was, however, not confirmed by our findings. Current-use of LD showed a non-significant negative association with FN-BMD and a non-significant positive association with LSBMD. Furthermore, the study of Rejnmark et al. in 2005 
showed also a non-significantly higher LS-BMD for the group with LD (who had been treated with a LD for at least 2 years prior to inclusion in the study), compared with nonusers [38]. Another study of Rejnmark et al. in 2006 showed a decrease in BMD for the LD users compared to placebo treated for 1 year. The effect was however weakened after 6 months, end of treatment. Unfortunately, we cannot confirm this result in our study. In our analysis of the association between the duration of LD and FN-BMD, we found a positive association for LD use between 1 and 365 days and a negative association with FN-BMD for longer use of LD (>365 days) compared to never-users of LD; nevertheless, this was not significant. Also we found an increase in LSBMD when LD was used for 121-365 days. This suggests that LD use might be weakly associated with higher BMD but only for a short time period.

Some studies have shown differences in vitamin $\mathrm{D}$ level according to LD use. In line with our results, there is evidence that $L D$ users have a lower serum $25(\mathrm{OH}) \mathrm{D}$ level than non-users [34] and the inverse association between LD use and serum $25(\mathrm{OH}) \mathrm{D}$ level has been reported before [16]. To the best of our knowledge, information about the effect modification by serum $25(\mathrm{OH}) \mathrm{D}$ level on the association between LD use and FN-BMD and LS-TBS as bone health parameters has not been reported earlier. In our study, stratified analyses did not show consistent patterns and the differences were not statistically significantly different between cut-offs of serum $25(\mathrm{OH}) \mathrm{D}$ level. However, we found evidence for potential effect modification $(P$-interaction $<0.10)$ by serum $25(\mathrm{OH}) \mathrm{D}$ level for the association of LD past-use and LSTBS. Stratified analyses showed a slightly stronger inverse association between past LD use and LS-TBS in those with serum 25(OH)D level $\leq 20$; however, the association was not significant in strata of serum 25(OH)D level between 20-50 and above $50 \mathrm{nmol} / \mathrm{L}$. This finding may imply that past-use of LD may have affected LS-TBS which can have a higher impact in those with extremely low $25(\mathrm{OH}) \mathrm{D}$ levels. However, because this effect modification was not further confirmed by other categories of LD use, further replication is needed. Moreover, even though we adjusted our analysis for body fat mass, reduced vitamin D levels may still be confounded by other measures of body composition and health status.

Our initial hypothesis was that an adequate serum $25(\mathrm{OH})$ D level and dietary calcium intake would counteract the potential adverse effects of LD on bone health. In contrast, we found no consistent significant differences between categories of dietary calcium intake, in the association between LD and FN-BMD, LS-BMD and LS-TBS. Thus, given that LD increases the renal excretion of calcium [40], the effects of LD on bone health showed unexpected results. This is in contrast with earlier studies, showing that a higher dietary intake of calcium prevented the expected decrease of
FN-BMD in LD users [38, 41, 42]. Potentially, the lack of calcium modifying the association between LD and bone health in our study may result from the fact that in our study calcium intake was higher than in other studies.

\section{Strengths and Limitations}

A strength in our study is the availability of data on dietary intake of calcium, serum $25(\mathrm{OH}) \mathrm{D}$ level and LD use, and the different indices of bone health (i.e. FN-BMD as well as LS-BMD and LS-TBS). Our study also adds data to the quite unexplored field of nutrient-drug interactions in a population of community-dwelling older persons, who are at risk of musculoskeletal diseases, malnutrition and consequences of polypharmacy. However, some limitations need to be taken into account when interpreting our results. These include the observational study design and potential residual confounding (for example, underlying disease), which prevents us from drawing conclusions regarding causality and the direction of the effect size of the association. Furthermore, as all our analyses were hypothesis-based, we did not adjust for multiple comparisons. With regard to possible type I errors, stringent interpretation of $p$ values should be made with caution. Likewise, potential misclassification of LD use using pharmacy records may occur because data regarding actual compliance was lacking. Also, dietary calcium intake was assessed using self-reported dietary assessment, which is subject to bias and measurement error. Also, dietary intake was measured only once (at baseline) and as a result we were unable to account for differences in dietary habits over time (e.g. due to disease or medication use). Moreover, the complete data on calcium or vitamin D supplement use was not available, which could lead to an underestimation of dietary intake. In addition, vitamin D and calcium prescriptions were not analysed as exposure variable because of confounding by indication. Also, we did not have a comprehensive assessment of calcium homeostasis. For example, the free calcium concentration in plasma is strongly controlled through a complicated physiological system including the interaction of calciotropic hormones such as PTH and 1,25(OH)D and only in extreme situations will the serum calcium concentration deflect from the normal range [14]. Since we did not have the availability of serum PTH, we could not investigate potential pathways between the use of LD and calcium intake and vitamin D level. Finally, our result of inconsistent effect modification by serum $25(\mathrm{OH})$ $\mathrm{D}$ and dietary calcium intake on the associations between $\mathrm{LD}$ and the parameters of bone health could be explained by managing the parathyroid hormones $(\mathrm{PTH})$ and $1.25(\mathrm{OH}) \mathrm{D}$ that maintain calcium homeostasis, especially when serum calcium is reduced [43].

In conclusion, this study does not support the hypothesis that the association between loop diuretic use and indices 
of bone health is modified by serum $25(\mathrm{OH}) \mathrm{D}$ level and calcium intake. However, because of polypharmacy effects and a higher risk of malnutrition in elderly, further research and replication is warranted on nutrient-drug interaction on bone health (considering the subjects with osteopenia and osteoporosis and people with malnutrition), using other biomarkers as PTH and bone turnover markers as well as long-term loop diuretic use.

Acknowledgements The input of inhabitants, general practitioners and pharmacists of the Ommoord district to the Rotterdam Study is appreciatively acknowledged.

Author Contributions Author's roles: SOA, JCKdJ, AGU, BHS and $\mathrm{NvdV}$ discussed the hypothesis and interpreted the data. KT, FK and FR provided the data. SOA and JKdJ analysed the data. AGU, BHS and MAI coordinated and directed the project. SOA, JCdJ, and NvdV wrote the article. SOA, JC KdJ, KT, FK, FR, MCZ, NMvS, LCGMdG, MAI, AGU, BHS and NvdV edited the article.

Funding The Rotterdam Study is supported by the Erasmus MC University Medical Center the Netherlands Organization for Scientific Research (NWO); The Netherlands Organization for Health Research and Development (ZonMw); the Research Institute for Diseases in the Elderly (RIDE); The Netherlands Genomics Initiative (NGI); the Ministry of Education, Culture and Science; the Ministry of Health, Welfare and Sports; the European Commission (DG XII); the Municipality of Rotterdam; and DSM Nutritional Products AG, Kaiseraugst, Switzerland provided funding for the assessment of vitamin D.

\section{Compliance with Ethical Standards}

Conflict of interest The authors declare no conflict of interest.

Human and Animal Rights and Informed Consent The study has been approved by the institutional review board (Medical Ethics Committee) of the Erasmus Medical Center and by the review board of the Netherlands Ministry of Health, Welfare and Sports, and with the 1964 Helsinki declaration and its later amendments and approval has been renewed every 5 years. From all subjects written informed consent was obtained.

Open Access This article is distributed under the terms of the Creative Commons Attribution 4.0 International License (http://creativeco mmons.org/licenses/by/4.0/), which permits unrestricted use, distribution, and reproduction in any medium, provided you give appropriate credit to the original author(s) and the source, provide a link to the Creative Commons license, and indicate if changes were made.

\section{References}

1. Otles S, Senturk A (2014) Food and drug interactions: a general review. Acta Sci Pol Technol Aliment 13(1):89-102

2. Gurwitz JH (1994) Suboptimal medication use in the elderly. The tip of the iceberg. JAMA 272(4):316-317

3. Beaulieu MD, Dufresne L, LeBlanc D (1998) Treating hypertension the right drugs given to the right patients? Can Fam Phys 44:294-298, 301-302
4. Sica DA, Gehr TWB, Frishman WH (2017) Use of diuretics in the treatment of heart failure in older adults. Heart Fail Clin 13(3):503-512

5. Xiao X, Xu Y, Wu Q (2018) Thiazide diuretic usage and risk of fracture: a meta-analysis of cohort studies. Osteoporos Int 29(7):1515-1524

6. Cumming RG, Klineberg RJ (1993) Psychotropics, thiazide diuretics and hip fractures in the elderly. Med J Aust 158(6):414-417

7. Rashiq S, Logan RF (1986) Role of drugs in fractures of the femoral neck. Br Med J (Clin Res Ed) 292(6524):861-863

8. Coe FL et al (1973) Evidence for secondary hyperparathyroidism in idiopathic hypercalciuria. J Clin Invest 52(1):134-142

9. Reichel $\mathrm{H}$ et al (1992) Frusemide therapy and intact parathyroid hormone plasma concentrations in chronic renal insufficiency. Nephrol Dial Transplant 7(1):8-15

10. Rejnmark L et al (2003) Dose-effect relations of loop- and thiazide-diuretics on calcium homeostasis: a randomized, doubleblinded Latin-square multiple cross-over study in postmenopausal osteopenic women. Eur J Clin Invest 33(1):41-50

11. Holm JP, Hyldstrup L, Jensen JB (2016) Time trends in osteoporosis risk factor profiles: a comparative analysis of risk factors, comorbidities, and medications over twelve years. Endocrine 54(1):241-255

12. Lim LS et al (2008) Loop diuretic use and increased rates of hip bone loss in older men: the Osteoporotic Fractures in Men Study. Arch Intern Med 168(7):735-740

13. Rejnmark L et al (2006) Loop diuretics increase bone turnover and decrease BMD in osteopenic postmenopausal women: results from a randomized controlled study with bumetanide. J Bone Miner Res 21(1):163-170

14. Cashman KD (2002) Calcium intake, calcium bioavailability and bone health. Br J Nutr 87(Suppl 2):S169-S177

15. Cashman KD (2007) Diet, nutrition, and bone health. J Nutr 137(11 Suppl):2507S-2512S

16. Sohl E et al (2012) The impact of medication on vitamin D status in older individuals. Eur J Endocrinol 166(3):477-485

17. van Orten-Luiten AC et al (2016) Vitamin D deficiency as adverse drug reaction? A cross-sectional study in Dutch geriatric outpatients. Eur J Clin Pharmacol 72(5):605-614

18. Fleet JC (2017) The role of vitamin D in the endocrinology controlling calcium homeostasis. Mol Cell Endocrinol 453:36-45

19. Ikram MA et al (2017) The Rotterdam Study: 2018 update on objectives, design and main results. Eur J Epidemiol 32(9):807-850

20. Klipstein-Grobusch K et al (1998) Dietary assessment in the elderly: validation of a semiquantitative food frequency questionnaire. Eur J Clin Nutr 52(8):588-596

21. Goldbohm RA et al (1994) Validation of a dietary questionnaire used in a large-scale prospective cohort study on diet and cancer. Eur J Clin Nutr 48(4):253-265

22. Voortman T et al (2017) Adherence to the 2015 Dutch dietary guidelines and risk of non-communicable diseases and mortality in the Rotterdam Study. Eur J Epidemiol 32(11):993-1005

23. Willett WC, Howe GR, Kushi LH (1997) Adjustment for total energy intake in epidemiologic studies. Am J Clin Nutr 65(4 Suppl):1220S-1228S discussion 1229S-1231S

24. Hofman A et al (2013) The Rotterdam Study: 2014 objectives and design update. Eur J Epidemiol 28(11):889-926

25. Holick MF (2007) Vitamin D deficiency. N Engl J Med 357(3):266-281

26. Ross AC et al (2011) The 2011 report on dietary reference intakes for calcium and vitamin D from the Institute of Medicine: what clinicians need to know. J Clin Endocrinol Metab 96(1):53-58 
27. Methodology, W.C.C.f.D.S. ATC/DDD Index 218. 2018. https:// www.whocc.no/atc_ddd_index/

28. Hofman A et al (2015) The Rotterdam Study: 2016 objectives and design update. Eur J Epidemiol 30(8):661-708

29. Hans D et al (2011) Correlations between trabecular bone score, measured using anteroposterior dual-energy X-ray absorptiometry acquisition, and 3-dimensional parameters of bone microarchitecture: an experimental study on human cadaver vertebrae. J Clin Densitom 14(3):302-312

30. Harvey NC et al (2015) Trabecular bone score (TBS) as a new complementary approach for osteoporosis evaluation in clinical practice. Bone 78:216-224

31. de Jonge EAL et al (2017) Dietary acid load, trabecular bone integrity, and mineral density in an ageing population: the Rotterdam study. Osteoporos Int 28(8):2357-2365

32. Hans D et al (2011) Bone microarchitecture assessed by TBS predicts osteoporotic fractures independent of bone density: the Manitoba study. J Bone Miner Res 26(11):2762-2769

33. Budtz-Jorgensen E et al (2007) Confounder selection in environmental epidemiology: assessment of health effects of prenatal mercury exposure. Ann Epidemiol 17(1):27-35

34. VanderWeele TJ, Shpitser I (2013) On the definition of a confounder. Ann Stat 41(1):196-220

35. Ott SM (2018) Cortical or trabecular bone: what's the difference? Am J Nephrol 47(6):373-375

36. Sterne JA et al (2009) Multiple imputation for missing data in epidemiological and clinical research: potential and pitfalls. BMJ $338: \mathrm{b} 2393$
37. Rubin DB, Schenker N (1991) Multiple imputation in healthcare databases: an overview and some applications. Stat Med 10(4):585-598

38. Rejnmark L et al (2005) Effects of long-term treatment with loop diuretics on bone mineral density, calcitropic hormones and bone turnover. J Intern Med 257(2):176-184

39. Swanson CM et al (2015) Associations of 25-hydroxyvitamin D and 1,25-dihydroxyvitamin $\mathrm{D}$ with bone mineral density, bone mineral density change, and incident nonvertebral fracture. J Bone Miner Res 30(8):1403-1413

40. Rejnmark L et al (2001) Effects of thiazide- and loop-diuretics, alone or in combination, on calcitropic hormones and biochemical bone markers: a randomized controlled study. J Intern Med 250(2):144-153

41. Ooms ME et al (1993) Determinants of bone mineral density and risk factors for osteoporosis in healthy elderly women. J Bone Miner Res 8(6):669-675

42. Lips P, van Schoor NM (2011) The effect of vitamin D on bone and osteoporosis. Best Pract Res Clin Endocrinol Metab 25(4):585-591

43. Veldurthy V et al (2016) Vitamin D, calcium homeostasis and aging. Bone Res 4:16041

Publisher's Note Springer Nature remains neutral with regard to jurisdictional claims in published maps and institutional affiliations. 